\title{
On the Double Doppler Effect Generated by Scatterer Motion
}

\author{
Viet-Ha Pham ${ }^{1}$, Mohamed Haj Taieb ${ }^{1}$, Jean-Yves Chouinard ${ }^{1}$, Sébastien Roy ${ }^{1}$, Huu-Tue Huynh ${ }^{2}$ \\ ${ }^{1}$ Department of Electrical and Computer Engineering, Université Laval, Québec, Canada \\ ${ }^{2}$ Bac Ha International University, Hanoi, Vietnam
}

Correspondence: Viet-Ha Pham, phamviet@gel.ulaval.ca

Manuscript communication: received 29 September 2010, accepted 16 December 2010

\begin{abstract}
In a time-varying transmission channel, the received signals are subject to frequency shifts due to the Doppler effect. The Doppler frequency is dependent on the carrier frequency and channel variation rate. In a fixed wireless channel, the channel variations are caused by scatterer motion. In this paper, we investigate analytically the Doppler effects generated by scatterer motion under different scatterer velocity distributions using the ring-of-scatterers geometric model. The proposed model considers Doppler frequency components caused by scatterer mobility to both received and reflected signals at each scatterer, and therefore is called the double Doppler model. The analytical curves are compared and statistically tested with several measurement results published in the literature. At low scatterer speeds, e.g., generated by moving foliage, the exponential velocity distribution is an appropriate model to describe the time-varying nature of the fixed wireless channels. The curve fitting results also show that our analytical model better approaches the empirical curves than the single Doppler model does. However, further investigation is still needed to find a suitable scatterer velocity distribution that closely describes the double Doppler effect in fast-variation fixed wireless channels, e.g., caused by passing vehicles.
\end{abstract}

Keywords- Double Doppler effect, Doppler power spectrum, scatterer motion, scatterer velocity distribution.

This work was partially presented in The IASTED International Conference on Wireless Communications, WC 2010, July 2010, Banff, AB, Canada.

\section{INTRODUCTION}

In wireless communications, the Doppler phenomenon is caused by the varying nature of the propagation environment [1,2]. The Doppler shift is mostly assumed to be generated by subscriber motion. A receiver traveling at a speed of $v \mathrm{~m} / \mathrm{s}$ will cause a Doppler shift $v=\frac{f_{c} v}{v_{p}} \cos \alpha$ [2] to carrier frequency $f_{c}$, where $v_{p}$ is the signal propagation velocity, and $\alpha$ is the angle between the receiver traveling direction and the receiver-transmitter axis. In a NLOS environment, if the directions of arrival at the subscriber are uniformly distributed over $[0,2 \pi)$ and if the multipath components arrive at the same time with equal power, the Doppler power spectral density, due to subscriber motion, follows Clarke's famous model and has the well-known U-shape [1, 3-5].

While Clarke's model is a popular tool to describe the Doppler phenomenon caused by subscriber mobility, we cannot use this model for fixed wireless channels. For fixed wireless communications applications, e.g., the Multichannel Multipoint Distribution Service (MMDS) system, even if the subscriber and the transmitter are stationary, the received signals still experience Doppler shifts. This phenomenon was studied empirically in [6-8] but no analytic models were presented to support the measurement results until the publication of [9] and [10]. In [9], the problem was studied analytically and a channel model for the indoor channel was proposed. In this model, the scatterers are assumed either stationary or mobile at a fixed velocity or at uniformly distributed velocities. While this model is suitable for indoor environments, where the scatterers are either stationary, e.g., furnitures and walls, or slowly mobile at similar velocities, e.g., pedestrians, it is invalid for outdoor channels, where mobile scatterers have different velocities.

Using a different approach, Roy et al. [10], assumed that the subscribers are moving at a constant speed while the scatterers are moving at random velocities with different velocity distributions. The classic ring-of-scatterer geometric model was considered. The Doppler shift of a NLOS multipath component, reflected from a scatterer, is the summation of two Doppler frequencies. The first component is caused by the scatterer mobility with respect to the transmitter and the second one is due to the receiver mobility relative to the scatterer. To validate the model, the measured Doppler power spectra presented in [7] were reproduced and fitted to the analytical model. The model validation shows that the scatterer velocity randomness is successfully addressed and incorporated in the model of Roy. However, the Doppler frequency component caused by the scatterer mobility with respect to the receiver was not taken into account.

In this paper, we extend the research results presented in [10] by investigating the double Doppler phenomenon generated by scatterer mobility itself in 
a fixed wireless channel using the ring-of-scatterers geometric model. In the proposed model, both Doppler frequency components, caused by the scatterer mobility with respect to the transmitter and the receiver to the received and reflected signals, respectively, at a scatterer, are addressed and investigated in depth. The analytical model is studied under different scatterer velocity distributions, including uniform, Gaussian, exponential and triangular distributions. The proposed model is validated through curve fitting and statistical analysis with empirical curves, reproduced from the results published in [6-8].

The remainder of the paper is organized as follows. Section 2 presents the calculation of double Doppler frequencies caused by scatterer mobility. In Section 3, the autocorrelation functions and the Doppler power spectra are derived for the ring-of-scatterer geometric model under different velocity distributions. Curve fitting and statistical tests are presented in Section 4. Section 5 concludes the paper.

\section{Doppler Shift Caused by Scatterer Motion}

Consider a fixed wireless propagation channel where transmitted signals are emitted from the transmitter, reflected by a scatterer and received at the receiver. As the scatterer is assumed to be moving with respect to the transmitter, the signals received at the scatterer from the transmitter experience a Doppler frequency shift $v_{\mathrm{TS}}$ given by:

$$
v_{\mathrm{TS}}=-\frac{f_{c} \times v_{\mathrm{Sx}}}{v_{p}} \cos \alpha_{\mathrm{TS}},
$$

where $v_{\mathrm{Sx}}$ is the scatterer velocity and $\alpha_{\mathrm{TS}}$ is the angle between the scatterer velocity vector, $\vec{v}_{S x}$, and the scatterer-transmitter axis.

The signal received at the scatterer is then reflected to the receiver. The carrier frequency of the reflected signal is $f_{\mathcal{c}}+v_{\mathrm{TS}}$. Due to the motion of the scatterer with respect to the receiver, the signals reflected from the scatterer and received at the receiver are affected by an additional Doppler shift $v_{\mathrm{SR}}$, calculated by:

$$
v_{\mathrm{SR}}=\frac{\left(f_{c}+v_{\mathrm{TS}}\right) \times v_{\mathrm{Sx}}}{v_{p}} \cos \alpha_{\mathrm{SR}},
$$

where $\alpha_{\mathrm{SR}}$ is the angle between the scatterer velocity vector and the scatterer-receiver axis.

The ratio between the scatterer speed, $v_{S x}$, and the signal propagation velocity, $v_{p}$, is very small, usually on the order of $10^{-7}$. Doppler frequency $v_{\mathrm{TS}}$ is on the order of tens of $\mathrm{Hz}$, and thus negligible compared to the carrier frequency, $f_{c}$. (2) is rewritten as follows:

$$
v_{\mathrm{SR}} \approx \frac{f_{c} \times v_{\mathrm{Sx}}}{v_{p}} \cos \alpha_{\mathrm{SR}} .
$$

The total Doppler shift caused by the scatterer motion to the signals received at the receiver is the sum of the

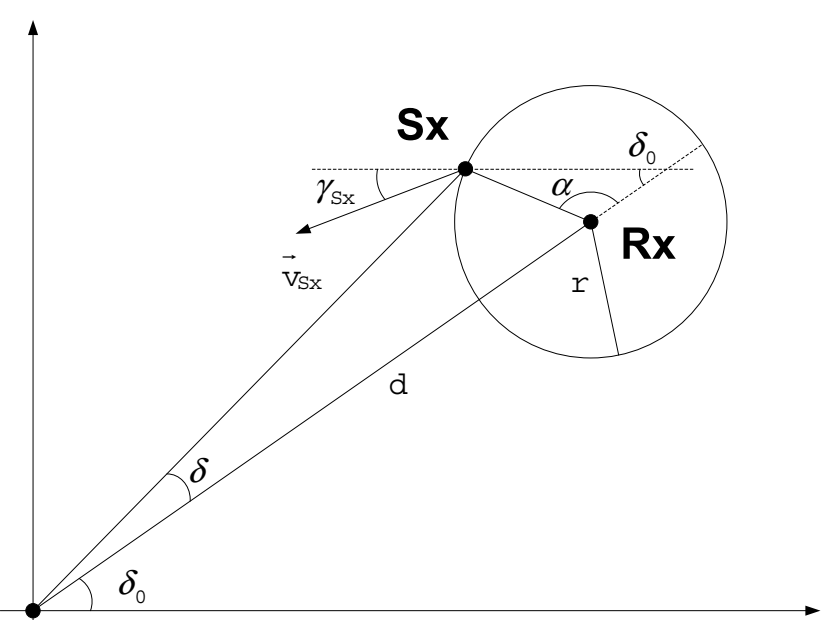

Tx

Figure 1. Geometric scenario of the scattering model [10].

two Doppler components, $v_{\mathrm{TS}}$ and $v_{\mathrm{SR}}$, calculated in (1) and (3), i.e.

$$
v_{S}=v_{\mathrm{TS}}+v_{\mathrm{SR}} \text {. }
$$

The Doppler shift caused by the scatterer motion with respect to the transmitter, $v_{\mathrm{TS}}$, was discussed and characterized in [10]. The double Doppler components, $v_{\mathrm{TS}}$ and $v_{\mathrm{SR}}$, were analytically investigated in [9] for indoor channels with mixed stationary and mobile scatterers at fixed velocities. The empirical Doppler power spectra caused by scatterer mobility in fixed wireless channels were presented in $[6-8,11,12]$. In the subsequent sections, we investigate the double Doppler effects for the fixed wireless channel with mobile scatterers under different scatterer velocity distributions.

\section{Doppler Power Spectral Density}

\subsection{Geometry Model}

We consider the ring-of-scatterers geometric model $[3,10]$ in order to investigate the double Doppler phenomenon. As illustrated in Figure 1, the transmitterreceiver separation is $d$. The mobile scatterers are uniformly distributed on a circle of radius $r$ around the receiver. In order to guarantee that the attenuation on each multipath component is independent of path length, the radius $r$ needs to be small in comparison to the distance $d$ : $r \ll d$. There is no direct component received at the receiver from the transmitter. The multipath components are supposed to have equal power and the multipath excess delay differences are assumed not greater than the time resolution of the receiver.

The transmitter-receiver axis forms an angle $\delta_{0}$ with the $x$ axis. The angle between the transmitter-receiver axis and the transmitter-scatterer axis is denoted $\delta$. Since the scatterers are uniformly distributed on a circle, the angle between the transmitter-receiver axis and the receiver-scatterer axis, $\alpha$, is uniformly distributed between 0 and $2 \pi$. The transmitter, $\mathrm{Tx}$, and the receiver, 
$\mathrm{Rx}$, are stationary. The scatterers, $\mathrm{Sx}$, are mobile. The direction $\gamma_{S x}$ of the scatterer velocity vectors is also uniformly distributed between 0 and $2 \pi$. The double Doppler effect will be investigated under different scatterer velocity distributions, including Gaussian, exponential, uniform and triangular distributions.

\subsection{Autocorrelation Function}

Following the same reasoning used in $[3,13,14]$, the received carrier from the $n$th scatterer is given by:

$$
s_{n}(t)=C_{n} \exp j\left(2 \pi\left(f_{c}+v_{n}\right) t+\theta_{n}\right),
$$

where $C_{n}$ is the attenuation; $v_{n}$ is the compound Doppler shift caused by transmitter, scatterer and receiver, if any, and $\theta_{n}$ is the total phase shift. These parameters are calculated on the transmitter-scattererreceiver path.

The received carrier at the receiver at passband is the summation of reflected signals from all scatterers, as follows:

$$
s(t)=\sum_{n=1}^{N} C_{n} \exp j\left(2 \pi\left(f_{c}+v_{n}\right) t+\theta_{n}\right),
$$

where $N$ is the number of mobile scatterers.

The baseband representation of the received carrier is:

$$
\begin{aligned}
c(t) & =\sum_{n=1}^{N} C_{n} \exp j\left(2 \pi v_{n} t+\theta_{n}\right) \\
& =T_{c}(t)+j T_{s}(t),
\end{aligned}
$$

where

$$
\begin{aligned}
& T_{\mathcal{C}}(t)=\sum_{n=1}^{N} C_{n} \cos \left(2 \pi v_{n} t+\theta_{n}\right), \\
& T_{S}(t)=\sum_{n=1}^{N} C_{n} \sin \left(2 \pi v_{n} t+\theta_{n}\right) .
\end{aligned}
$$

The autocorrelation of the received carrier at baseband is:

$$
\begin{aligned}
R(\tau) & =\left\langle c(t) c^{*}(t-\tau)\right\rangle \\
& =\left\langle\left(T_{c}(t)+j T_{s}(t)\right)\left(T_{c}(t-\tau)-j T_{s}(t-\tau)\right)\right\rangle,
\end{aligned}
$$

where $\langle\cdot\rangle$ is the averaging operator.

Following [14], pp. 77-78, the $\left\langle\left(T_{\mathcal{c}}(t) T_{\mathcal{c}}(t-\tau)\right\rangle\right.$ term can be expressed as:

$$
\begin{aligned}
\left\langle\left(T_{\mathcal{C}}(t) T_{\mathcal{C}}(t-\tau)\right\rangle\right. & \left.=\left\langle T_{\mathcal{S}}(t) T_{\mathcal{S}}(t-\tau)\right)\right\rangle \\
\left\langle\left(T_{\mathcal{C}}(t) T_{\mathcal{S}}(t-\tau)\right\rangle\right. & \left.=-\left\langle T_{\mathcal{S}}(t) T_{\mathcal{c}}(t-\tau)\right)\right\rangle .
\end{aligned}
$$

Replacing (10) in (9), the autocorrelation $R(\tau)$ is:

$$
\begin{aligned}
R(\tau)= & 2\left\langle\left(T_{\mathcal{c}}(t) T_{\mathcal{c}}(t-\tau)\right\rangle-2 j\left\langle\left(T_{\mathcal{c}}(t) T_{\mathcal{S}}(t-\tau)\right)\right\rangle\right. \\
= & 2\left[\frac{1}{2} E_{0}^{2} \sum_{n=1}^{N}\left\langle\cos \left(-2 \pi v_{n} \tau\right)\right\rangle\right. \\
& \left.-j \frac{1}{2} E_{0}^{2} \sum_{n=1}^{N}\left\langle\sin \left(-2 \pi v_{n} \tau\right)\right\rangle\right] \\
= & E_{0}^{2} \sum_{n=1}^{N}\left\langle e^{j 2 \pi v_{n} \tau}\right\rangle
\end{aligned}
$$

where $E_{0}$ is the signal magnitude, i.e., square root of the signal power: $E_{0}^{2}=\sum_{n=1}^{N} C_{n}^{2}$.

When $N$ tends towards infinity, the summation in (9) becomes an integral on the circle of radius $r$, i.e., on the continuous variable $\alpha$ from 0 to $2 \pi$ :

$$
R(\tau)=\frac{1}{2 \pi} E_{0}^{2} \int_{0}^{2 \pi}\left\langle e^{j 2 \pi v \tau}\right\rangle d \alpha
$$

Replacing the corresponding Doppler components, $v$, into (12), we obtain the autocorrelation function, and therefore, the Doppler power spectrum ${ }^{1}$.

\subsection{Doppler Spectra Generated by Scatterer Motion}

Utilizing the ring-of-scatterers geometric model in Figure 1, the angles $\alpha_{\mathrm{TS}}$ and $\alpha_{\mathrm{SR}}$ are derived as:

$$
\begin{aligned}
& \alpha_{\mathrm{TS}}=\delta_{0}+\delta-\gamma_{\mathrm{Sx}} \\
& \alpha_{\mathrm{SR}}=\alpha+\delta_{0}-\gamma_{\mathrm{Sx}} .
\end{aligned}
$$

The Doppler frequency caused by scatterer motion is:

$$
\begin{aligned}
v_{\mathrm{S}} & =v_{\mathrm{TS}}+v_{\mathrm{SR}} \\
& =\frac{f_{c} v_{\mathrm{Sx}}}{v_{p}}\left[\cos \left(\delta_{0}+\delta-\gamma_{\mathrm{Sx}}\right)+\cos \left(\alpha+\delta_{0}-\gamma_{\mathrm{Sx}}\right)\right] \\
& =2 \frac{f_{c} v_{\mathrm{Sx}}}{v_{p}} \cos \left(\frac{\delta+\alpha}{2}+\delta_{0}-\gamma_{\mathrm{Sx}}\right) \cos \left(\frac{\delta-\alpha}{2}\right) .
\end{aligned}
$$

The autocorrelation function of the received carrier complex envelope is represented in (15). Reducing the integral on variable $\gamma_{S x}$ gives:

$$
\begin{aligned}
& R(\tau)= \\
& \quad \frac{1}{2 \pi} E_{0}^{2} \int_{v_{S \mathrm{~S}}} \int_{0}^{2 \pi} J_{0}\left(4 \pi \frac{f_{c} v_{S \mathrm{x}}}{v_{p}} \cos \left(\frac{\delta-\alpha}{2}\right) \tau\right) \\
& p_{v_{\mathrm{Sx}}}\left(v_{\mathrm{Sx}}\right) d \alpha d v_{\mathrm{Sx}} .
\end{aligned}
$$

Next, the integral on variable $\alpha$ is reduced. One observes that, as $\alpha$ varies from 0 to $2 \pi, \frac{\delta-\alpha}{2}$ varies from 0 to $\pi$. Applying (6.681.5) in [19], pp. 724 gives:

$$
R(\tau)=\int_{v_{\mathrm{Sx}}} J_{0}^{2}\left(2 \pi \frac{f_{c} v_{\mathrm{Sx}}}{v_{p}} \tau\right) p_{v_{\mathrm{Sx}}}\left(v_{\mathrm{Sx}}\right) d v_{\mathrm{Sx}} .
$$

One observes that the autocorrelation, and therefore the Doppler spectrum, is dependent on the scatterer velocity distribution. If the scatterer velocity is constant, i.e., $v_{\mathrm{Sx}}=v_{\max }$, performing the Fourier transform on the above autocorrelation function, one obtains:

$$
S(v)=\frac{1}{2 \pi v_{\text {Smax }}} G_{22}^{20}\left(\begin{array}{c|c}
v^{2} & \frac{1}{2}, \frac{1}{2} \\
4 v_{\text {Smax }}^{2} & 0,0
\end{array}\right),
$$

where $G_{p q}^{m n}\left(x \mid \begin{array}{l}a_{1}, \cdots, a_{n}, a_{n+1}, \cdots, a_{p} \\ b_{1}, \cdots, b_{m}, b_{m+1}, \cdots, b_{q}\end{array}\right)$ represents the MeijerG function and $v_{\text {Smax }}$ is the maximum

${ }^{1}$ To reproduce our results, it is important to note that, the autocorrelation functions are calculated manually, using the integrals found in [15-18], whereas the Doppler spectra are calculated with the help of the Maple computing software. However, since it cannot perform the integrals with complex exponentials, we decomposed the Fourier transforms into two integrals, corresponding to the real and the imaginary parts. Each integral is calculated separately by Maple and then, combined to obtain the final results presented in this section. 


$$
\begin{aligned}
R(\tau) & =\frac{1}{2 \pi} E_{0}^{2} \int_{0}^{2 \pi}\left\langle\exp \left[j 2 \pi 2 \frac{f_{c} v_{\mathrm{Sx}}}{v_{p}} \cos \left(\frac{\delta+\alpha}{2}+\delta_{0}-\gamma_{\mathrm{Sx}}\right) \cos \left(\frac{\delta-\alpha}{2}\right) \tau\right]\right\rangle d \alpha \\
& =\frac{1}{2 \pi} E_{0}^{2} \int_{\alpha} \int_{\gamma_{\mathrm{Sx}}} \int_{v_{\mathrm{Sx}}} \exp \left[j 4 \pi \frac{f_{c_{\mathcal{S}} v_{\mathrm{Sx}}}}{v_{p}} \cos \left(\frac{\delta+\alpha}{2}+\delta_{0}-\gamma_{\mathrm{Sx}}\right) \cos \left(\frac{\delta-\alpha}{2}\right) \tau\right] p_{\gamma_{\mathrm{Sx}}}\left(\gamma_{\mathrm{Sx}_{\mathrm{x}}}\right) p_{v_{\mathrm{Sx}}}\left(v_{\mathrm{Sx}}\right) d \alpha d \gamma_{\mathrm{Sx}_{\mathrm{x}}} d v_{\mathrm{Sx}} \\
& =\frac{1}{(2 \pi)^{2}} E_{0}^{2} \int_{v_{\mathrm{Sx}}} \int_{\alpha} \int_{\gamma_{\mathrm{Sx}}} \exp \left[j 4 \pi \frac{f_{c} v_{\mathrm{Sx}}}{v_{p}} \cos \left(\frac{\delta+\alpha}{2}+\delta_{0}-\gamma_{\mathrm{Sx}}\right) \cos \left(\frac{\delta-\alpha}{2}\right) \tau\right] p_{v_{\mathrm{Sx}}}\left(v_{\mathrm{Sx}}\right) d \gamma_{\mathrm{Sx}} d \alpha d v_{\mathrm{Sx}}
\end{aligned}
$$

Doppler frequency induced by scatterer motion on the reflected signal, i.e., $v_{\mathrm{Smax}}=\frac{f_{c} v_{\max }}{v_{p}}$.

In the following Sections, we analytically derive the autocorrelation functions and the Doppler power spectral densities for uniform, exponential, Gaussian and triangular scatterer velocity.

3.3.1 Uniform Distribution: The scatterer velocity is uniformly distributed from 0 to $v_{\max }$. The probability density function (pdf) is:

$$
p\left(v_{\mathrm{Sx}}\right)= \begin{cases}\frac{1}{v_{\max }}, & 0 \leq v_{\mathrm{Sx}} \leq v_{\max } \\ 0, & \text { otherwise }\end{cases}
$$

where $\mu_{v_{S x}}=\frac{v_{\max }}{2}$ and $\sigma_{v_{S x}}=\frac{v_{\max }}{2 \sqrt{3}}$ are the mean and standard deviation of the scatterer velocity, respectively. The autocorrelation function then becomes:

$$
\begin{aligned}
R(\tau) & =\frac{1}{v_{\max }} \int_{0}^{v_{\max }} J_{0}^{2}\left(\frac{2 \pi f_{c}}{v_{p}} v_{\mathrm{Sx}_{\mathrm{x}}} \tau\right) d v_{\mathrm{Sx}} \\
& ={ }_{2} F_{3}\left(\frac{1}{2}, \frac{1}{2} ; 1,1, \frac{3}{2} ;-\left(\frac{2 \pi f_{\mathcal{c}} v_{\max }}{v_{p}} \tau\right)^{2}\right) \\
& ={ }_{2} F_{3}\left(\frac{1}{2}, \frac{1}{2} ; 1,1, \frac{3}{2} ;-\left(2 \pi v_{\mathrm{S} \max } \tau\right)^{2}\right),
\end{aligned}
$$

where ${ }_{p} F_{q}\left(a_{1}, \cdots, a_{p} ; b_{1}, \cdots, b_{q} ; x\right)$ is the generalized hypergeometric function. The corresponding Doppler power spectrum is:

$$
S(v)=\frac{G_{33}^{30}\left(\frac{v^{2}}{4 v_{\text {Smax }}^{2}} \mid \begin{array}{c}
\frac{1}{2}, \frac{1}{2}, 1 \\
0,0,0
\end{array}\right)}{4 \pi v_{\text {Smax }}} .
$$

3.3.2 Exponential distribution: The scatterer velocity is exponentially distributed with mean velocity $v_{0}$ and the pdf is:

$$
p\left(v_{\mathrm{Sx}}\right)=\left\{\begin{array}{lc}
\frac{1}{v_{0}} e^{-\frac{v_{\mathrm{Sx}}}{v_{0}},} & v_{\mathrm{Sx}} \geq 0, \\
0, & \text { otherwise, }
\end{array}\right.
$$

where $\mu_{v_{S \mathrm{X}}}=v_{0}$ and $\sigma_{v_{\text {Sx }}}=v_{0}$. The corresponding autocorrelation function is:

$$
\begin{aligned}
R(\tau) & =\frac{1}{v_{0}} \int_{0}^{\infty} J_{0}^{2}\left(\frac{2 \pi f_{c}}{v_{p}} v_{\mathrm{Sx}} \tau\right) e^{-\frac{v_{S \mathrm{~S}}}{v_{0}}} d v_{\mathrm{Sx}} \\
& =\frac{v_{p} G_{22}^{21}\left(\frac{v_{p}^{2}}{16 v_{0}^{2} \pi^{2} f_{c}^{2} \tau^{2}} \mid \begin{array}{c}
\frac{1}{2}, \frac{1}{2} \\
0,0
\end{array}\right)}{4 \pi^{2} v_{0} f_{c}|\tau|} \\
& =\frac{G_{22}^{21}\left(\frac{1}{16 \pi^{2} v_{0}^{2} \tau^{2}} \mid \begin{array}{l}
\frac{1}{2}, \frac{1}{2} \\
0,0
\end{array}\right)}{4 \pi^{2} v_{0}|\tau|}
\end{aligned}
$$

where $v_{0}$ is the maximum Doppler frequency caused by the scatterer motion at an average velocity of $v_{0}$ on its received signal, i.e., $v_{0}=\frac{f_{c} v_{0}}{v_{p}}$. Applying the Fourier transform on the autocorrelation function results in the following Doppler power spectrum:

$$
S(v)=\frac{K_{o}\left(\frac{|v|}{4 v_{0}}\right)^{2}}{2 \pi^{2} v_{0}}
$$

where $K_{0}(\cdot)$ is the zeroth order modified Bessel function of the second kind.

3.3.3 Gaussian distribution: Since the velocity (magnitude) is always positive or equal to zero, we use a half-Gaussian distribution for the calculation of the autocorrelation function. In order to keep the cumulative distribution function (cdf) at $\infty$ equal to 1 , a factor of 2 is introduced in the original distribution, thus yielding the scatterer velocity pdf

$$
p\left(v_{\mathrm{Sx}}\right)= \begin{cases}\frac{2}{\sqrt{2 \pi} \sigma} e^{-\frac{v_{\mathrm{SX}}^{2}}{\sigma^{2}},} & v_{\mathrm{Sx}} \geq 0 \\ 0, & \text { otherwise }\end{cases}
$$

where $\mu_{v_{S x}}=\sqrt{\frac{2}{\pi}} \sigma, \sigma_{v_{S x}}=\sqrt{\frac{\pi-2}{\pi}} \sigma$ and $\sigma^{2}$ is the variance parameter of the original Gaussian distribution. The autocorrelation function is computed as:

$$
\begin{aligned}
R(\tau) & =\frac{2}{\sqrt{2 \pi} \sigma} \int_{0}^{\infty} J_{0}^{2}\left(\frac{2 \pi f_{c}}{v_{p}} v_{\mathrm{Sx}} \tau\right) e^{-\frac{v_{\mathrm{Sx}}^{2}}{2 \sigma^{2}}} d v_{\mathrm{Sx}} \\
& ={ }_{2} F_{2}\left(\frac{1}{2}, \frac{1}{2} ; 1,1 ;-\frac{8 \pi^{2} f_{c}^{2}}{v_{p}^{2}} \sigma^{2} \tau^{2}\right) \\
& ={ }_{2} F_{2}\left(\frac{1}{2}, \frac{1}{2} ; 1,1 ;-\frac{8 \pi^{2}}{\lambda_{c}^{2}} \sigma^{2} \tau^{2}\right)
\end{aligned}
$$

with $\lambda_{c}$ being the carrier wavelength. The corresponding Doppler power spectrum is:

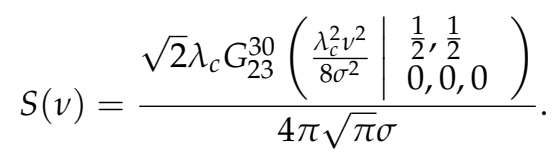

3.3.4 Triangular distribution: Here, the scatterer velocity distribution is given by:

$$
p\left(v_{\mathrm{Sx}}\right)=\left\{\begin{array}{l}
\frac{2}{v_{\max }}-\frac{2 v_{\mathrm{Sx}}}{v_{\max }^{2},} \quad 0 \leq v_{\mathrm{Sx}} \leq v_{\max }, \\
0, \quad \text { otherwise, }
\end{array}\right.
$$


whith $\mu_{v_{S x}}=\frac{v_{\max }}{3}$ and $\sigma_{v_{S x}}=\frac{v_{\max }}{3 \sqrt{2}}$. In this case, the autocorrelation function is given by:

$$
\begin{aligned}
R(\tau) & =\int_{0}^{v_{\max }} J_{0}^{2}\left(\frac{2 \pi f_{c}}{v_{p}} v_{\mathrm{Sx}} \tau\right)\left(\frac{2}{v_{\max }}-\frac{2 v_{\mathrm{Sx}}}{v_{\max }^{2}}\right) d v_{\mathrm{Sx}} \\
& =2_{2} F_{3}\left(\frac{1}{2}, \frac{1}{2} ; 1,1, \frac{3}{2} ;-\frac{4 \pi^{2} f_{c}^{2} v_{\max }^{2}}{v_{p}^{2}} \tau^{2}\right) \\
& -J_{0}\left(\frac{2 \pi f_{c} v_{\max }}{v_{p}} \tau\right)^{2}-J_{1}\left(\frac{2 \pi f_{c} v_{\max }}{v_{p}} \tau\right)^{2} \\
& =2_{2} F_{3}\left(\frac{1}{2}, \frac{1}{2} ; 1,1, \frac{3}{2} ;-4 \pi^{2} v_{\mathrm{S} \max }^{2} \tau^{2}\right) \\
& -J_{0}\left(2 \pi v_{\mathrm{Smax}} \tau\right)^{2}-J_{1}\left(2 \pi v_{\mathrm{S} \max } \tau\right)^{2} .
\end{aligned}
$$

Applying the Fourier transform on the autocorrelation function in (29), the Doppler power spectrum is obtained as

$$
\begin{aligned}
S(v)= & \frac{G_{33}^{30}\left(\frac{v^{2}}{4 v_{\text {Smax }}^{2}} \mid \begin{array}{l}
\frac{1}{2}, \frac{1}{2}, 1 \\
0,0,0
\end{array}\right)}{2 \pi v_{\text {Smax }}} \\
& -\frac{G_{22}^{20}\left(\frac{v^{2}}{4 v_{\text {Smax }}^{2}} \mid \begin{array}{l}
\frac{1}{2}, \frac{1}{2} \\
0,0
\end{array}\right)}{2 \pi v_{S \max }} . \\
& -\frac{G_{33}^{21}\left(\frac{v^{2}}{4 v_{\text {Smax }}^{2}} \mid \begin{array}{l}
-\frac{1}{2}, \frac{1}{2}, \frac{3}{2} \\
0,0, \frac{1}{2}
\end{array}\right)}{2 \pi v_{\text {Smax }}} .
\end{aligned}
$$

Figures 2(a) and 2(b) show the autocorrelation functions and the Doppler power spectra, respectively, for the above four scatterer velocity distributions: uniform, exponential, half-Gaussian and triangular distributions. In order to make the autocorrelation functions and the Doppler power spectra under the four scatterer velocity distributions comparable, the distribution parameters are selected so that the mean scatterer velocities, $\mu_{v_{S x} \text {, }}$ are equal to $1 \mathrm{~m} / \mathrm{s}$. The maximum scatterer velocity, $v_{\text {max }}$, is set to $2 \mathrm{~m} / \mathrm{s}$ for the uniform distribution and to $3 \mathrm{~m} / \mathrm{s}$ for the triangular distribution. The mean scatterer velocity for the exponential distribution, $v_{0}$, is $1 \mathrm{~m} / \mathrm{s}$. The variance parameter of the half-Gaussian distribution, $\sigma^{2}$, is set to $\frac{\pi}{2}$, leading to an average scatterer velocity of $1 \mathrm{~m} / \mathrm{s}$. The carrier frequency, $f_{c}$, is selected to be $300 \mathrm{MHz}$.

In Figure 2(b), it is observed that the the Doppler power spectrum is bounded under the uniform and triangular distributions but not under the exponential and half-Gaussian distributions. In this figure, the Doppler frequency is depicted between -7 and $7 \mathrm{~Hz}$. If the Doppler frequency range is increased, then the Doppler power spectrum continues to expand under the exponential and Gaussian distributions whereas they do not change under the uniform and rectangular distributions. This is because the scatterer velocity is bounded by the maximum velocity, $v_{\max }$, under the uniform and triangular distributions while it is not bounded under the exponential and half-Gaussian distributions.

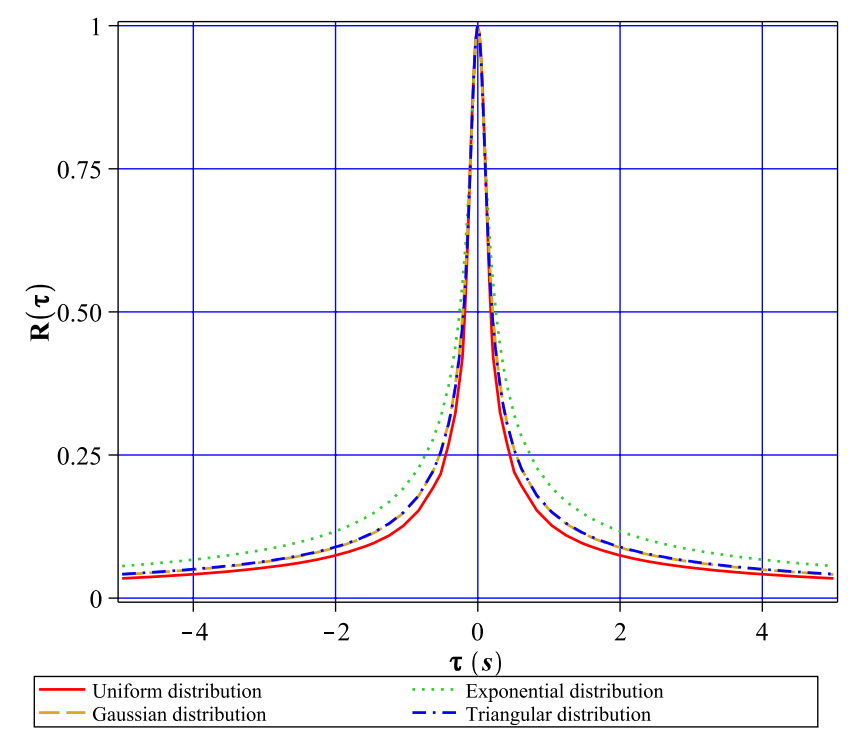

(a)

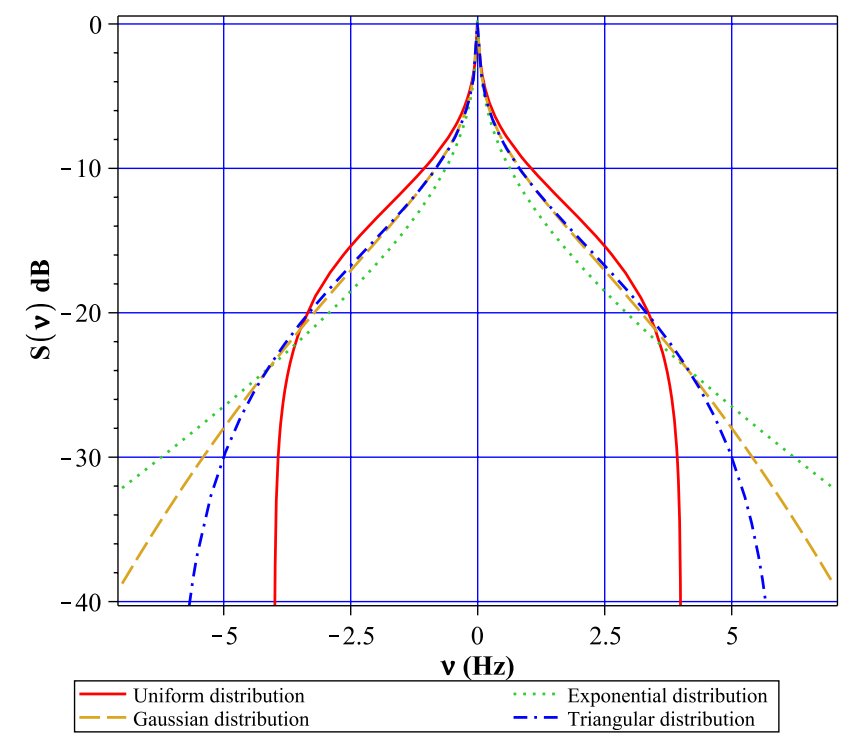

(b)

Figure 2. Autocorrelation function (a) and Doppler power spectrum (b) when the receiver and the transmitter are fixed, the scatterer velocity has different distributions.

\section{Model Validation}

The proposed model is validated through curve-fitting and statistical testing of the empirical Doppler power spectrum curves reproduced from [6-8], and our analytical curves. The analytical curves are also compared with those from [10] in order to show the improvement of the double Doppler model comparing with the single Doppler model.

In Figure 3, the empirical curve-fitting obtained with our model are compared to those presented in [10]. The empirical curves, measured at a carrier frequency of $2.5 \mathrm{GHz}$, were extracted and reproduced from [7, Figure 7]. The difference between the two models is the introduction in our model of the double Doppler components, caused by scatterer motion with respect to the transmitter and the receiver to the reflected signals. 


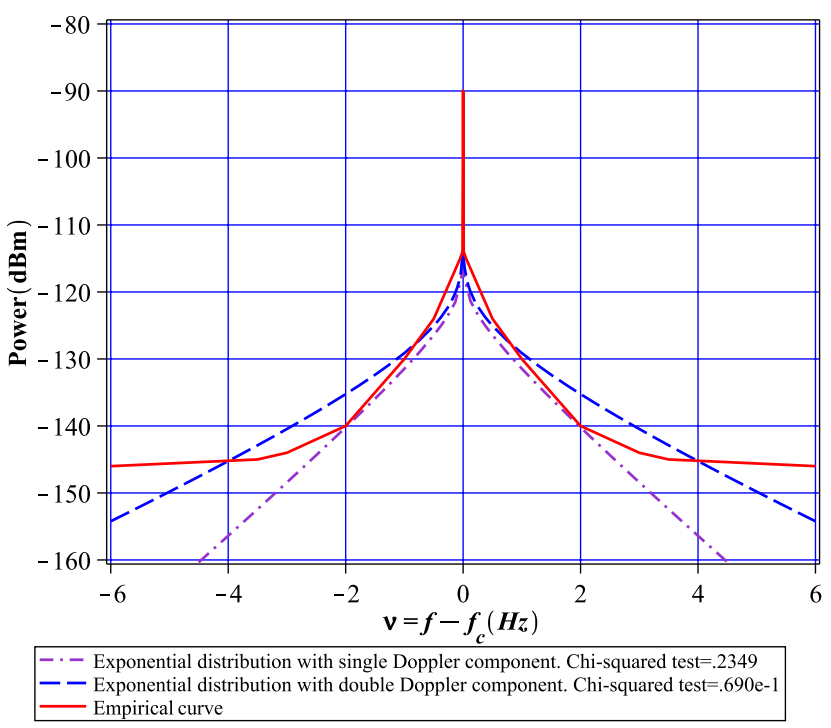

(a)

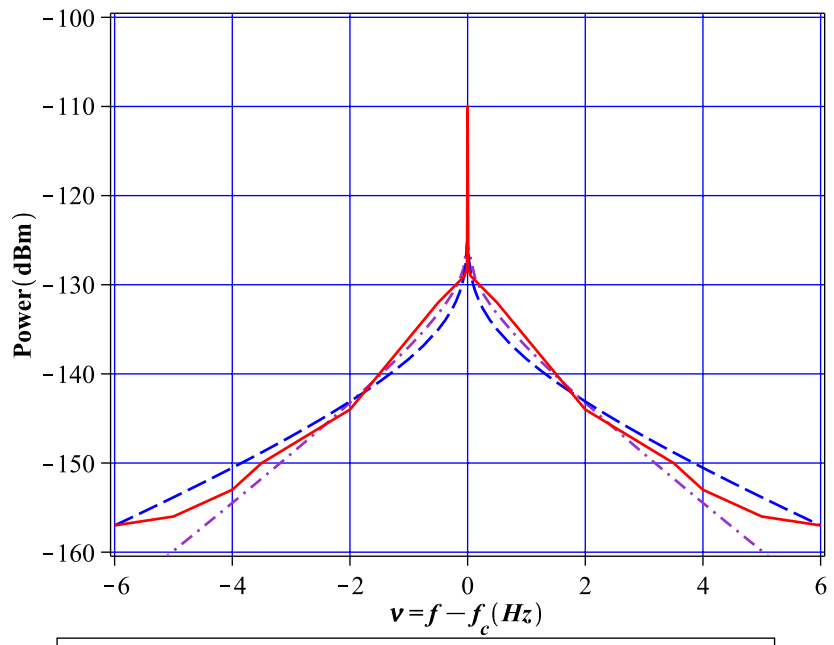

$-\cdot-$ Exponential distribution with single Doppler component. Chi-squared test $=.620$ - Exponential distribution with double Doppler component. Chi-squared test=.246 (b)

Figure 3. Performance comparison with the results presented in [10, Figures 10 and 11]: curve-fitting to the empirical Doppler power spectrum at (a) moderate and (b) high Doppler spread.

Figure 3(a) shows a Doppler power spectrum measured in a channel with moderate Doppler spread, while Figure 3(b) shows a Doppler power spectrum measured in an environment with high Doppler spread. The analytical curves are produced using an exponential scatterer velocity distribution. This distribution is selected because it provides the best curve fitting, among the four scatterer velocity distributions aforementioned, with the empirical curves. Our model tends to spread widely and thus better follow the measurement curves, whereas the model of Roy tends to descend more steeply and to deviate from the measurement curves. The Chi-square test results show that our model outperforms the model of Roy in both moderate and high Doppler spread channels.

Figure 4 compares the proposed analytical model curves with the empirical curves reproduced from [6, Figure 4]. Figure 4(a) shows the Doppler power spectra

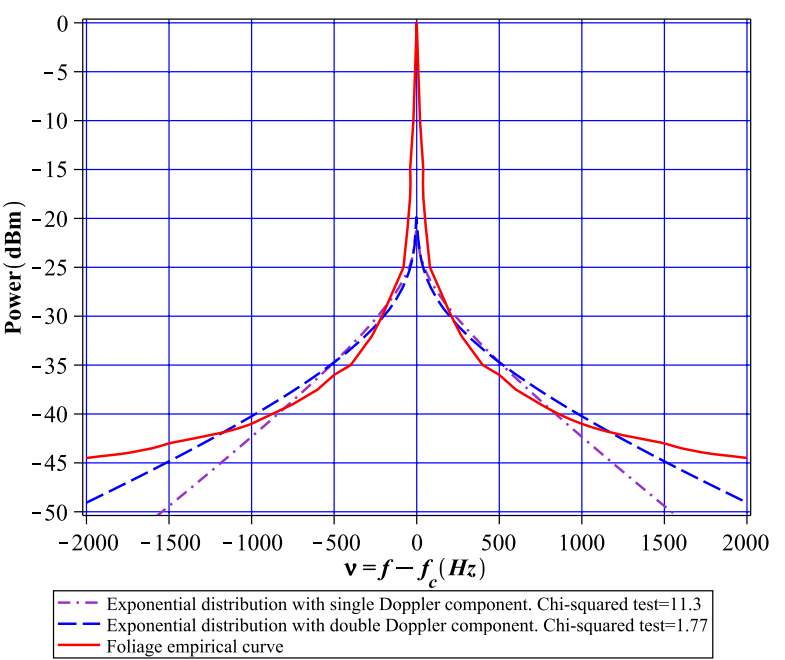

(a)

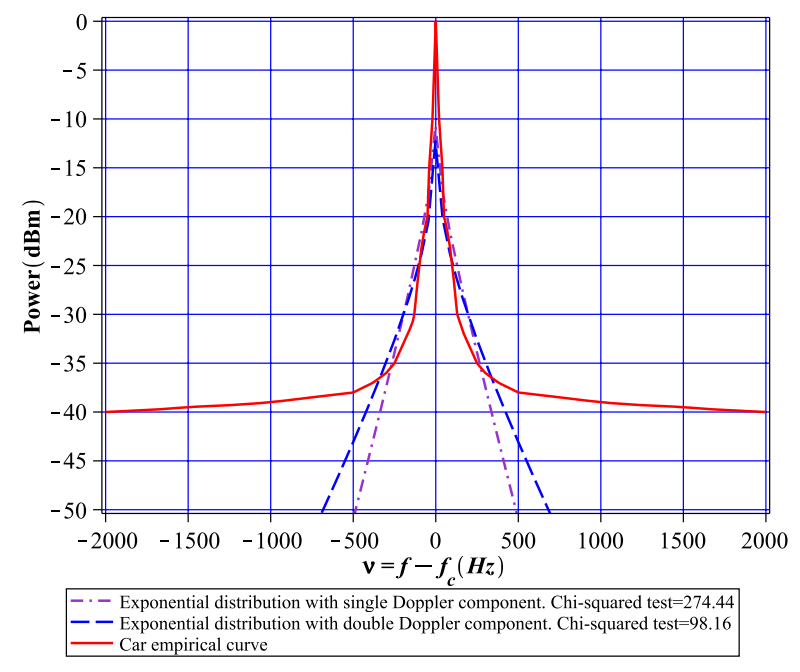

(b)

Figure 4. Curve fitting with the empirical results (extracted from [6, Figure 4]) generated by (a) foliage and by (b) passing vehicles.

generated by foliage movements, while Figure 4(b) represents the Doppler power spectra caused by passing vehicles, both in a fixed wireless channel at a carrier frequency of $29.5 \mathrm{GHz}$. The analytical curves for the model presented in [10] and our model are plotted under an exponential scatterer velocity distribution. It shows that our model outperforms the other model in both cases. However, while they follow relatively closely the Doppler power spectrum caused by foliage motion, neither of the two models follow the high Doppler shifts in the case of passing vehicles. Intuitively, the foliage fluctuates randomly in every direction due to the wind. Its velocity is slow and its average is close to zero. The exponential distribution also emphasizes the low speeds and therefore better describes the case of foliage motion than the case of vehicles moving at high speeds. It is conjectured that a Laplacian distribution would better describe the vehicle velocity distribution. Unfortunately, the calculation of the autocorrelation function and the Doppler power spectrum under the Laplacian distribution is currently 


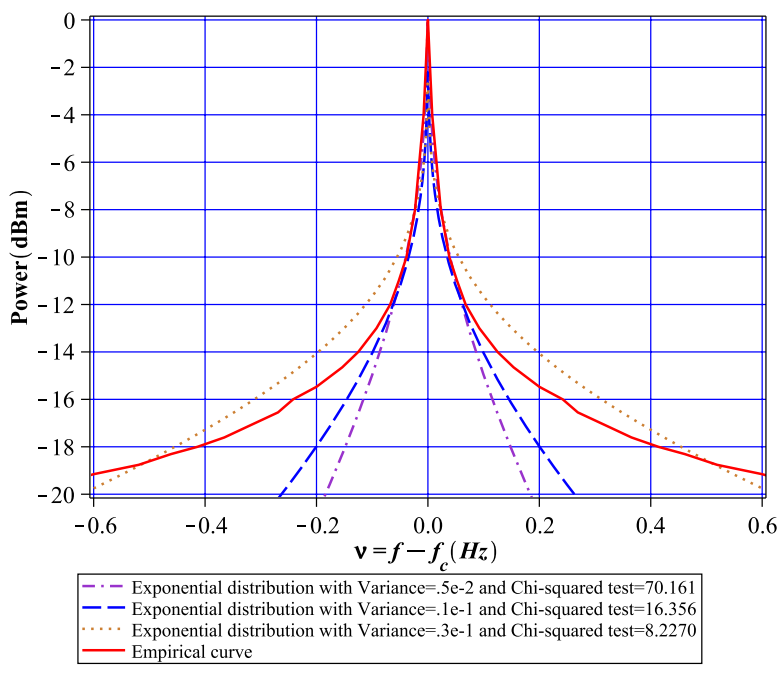

Figure 5. Curve fitting with the empirical results presented in [8, Figure 2]

difficult to handle, and therefore, no analytical results for the Laplacian scatterer velocity distribution is presented at this point.

Figure 5 shows the curve fitting results for the empirical curves presented in [8, Figure 2]. The measurements were conducted for a short-range transmission at 5.3 GHz. Three exponential distributions of the scatterer velocity with different variances are used in the tests. Once again, the Chi-square statistical test shows that the Doppler power spectrum under exponential scatterer velocity distribution follows closely the measurement curve and describes well the slow time-varying nature of the short-range channel at $5.3 \mathrm{GHz}$.

\section{Conclusions}

In this paper, the double Doppler effects caused by scatterer motion with respect to the transmitter and the receiver are studied under different scatterer velocity distributions, including uniform, exponential, half-Gaussian and triangular distributions. Analytical curves of the Doppler power spectrum are compared and statistically tested with measurement results, extracted and reproduced from empirical results published in the literature. The curve-fitting and statistical test results show that the exponential scatterer velocity distribution outperforms the other distributions and describes accurately the slow time-varying nature of the fixed wireless channels. It also shows that the proposed double Doppler model characterizes better the Doppler effects caused by scatterer mobility at low velocities than the single Doppler model does. In addition, more investigation is needed to find a suitable distribution that closely describes the scatterer velocities in fastvarying fixed wireless channels, e.g., caused by passing vehicles.

\section{ACKNOWLEDGEMENTS}

This work was supported by the Fonds québécois de la recherche sur la nature et les technologies (FQRNT) under research team project grant PR-113970 and by the Natural Sciences and Engineering Research Council of Canada (NSERC) under discovery grant RGPIN 42906.

\section{REFERENCES}

[1] W. C. Jakes, Microwave Mobile Communications. Wiley, 1974.

[2] T. S. Rappaport, Wireless Communications Principles and Practice, 2nd ed. Prentice Hall, 2002.

[3] M. J. Gans, "A power-spectral theory of propagation in the mobile-radio environment," IEEE Transaction on Vehicular Technology, vol. VT-21, no. 1, pp. 27-38, Feb. 1972.

[4] J. I. Smith, "A computer generated multipath fading simulation for mobile radio," IEEE Transactions on Vehicular Technology, vol. VT-24, no. 3, pp. 39-40, Aug. 1975.

[5] R. Narasimhan and D. C. Cox, "A generalized doppler power spectrum for wireless environments," IEEE Communications Letters, vol. 3, no. 6, pp. 164-165, June 1999.

[6] N. Naz and D. D. Falconer, "Temporal variations characterizations for fixed wireless at $29.5 \mathrm{GHz}$," in Proc. IEEE Vehicular Technology Conference (VTC), vol. 3, May 2000, pp. 2178-2182.

[7] D. S. Baum, D. Gore, R. Nabar, S. Panchanathan, K. V. S. Hari, V. Erceg, and A. J. Paulraj, "Measurement and characterization of broadband MIMO fixed wireless channels at $2.5 \mathrm{GHz}$," IEEE Int. Conf. Personal Wireless Communications, pp. 203-206, Dec. 2000.

[8] A. Domazetovic, L. J. Greenstein, N. B. Mandayam, and I. Seskar, "Estimating the Doppler spectrum of a shortrange fixed wireless channel," IEEE Communications Letters, vol. 7, no. 5, pp. 227-229, May 2003.

[9] S. Thoen, L. V. der Perre, and M. Engels, "Modeling the channel time-variance for fixed wireless communications," IEEE Communications Letters, vol. 6, no. 8, pp. 331-333, Aug. 2002.

[10] S. Roy, H. T. Huynh, and P. Fortier, "Compound Doppler spread effects of subscriber motion and scatterer motion," International Journal of Electronics and Communications, vol. 57, no. 4, pp. 237-246, 2003.

[11] S. J. Howard and K. Pahlavan, "Doppler spread measurements of indoor radio channel," IEEE Electronics Letters, vol. 26, no. 2, pp. 107-109, Jan. 1990.

[12] R. J. C. Bultitude, R. F. Hahn, and R. J. Davies, “Propagation considerations for the design of an indoor broadband communications system at EHF," IEEE Transactions on Vehicular Technology, vol. 47, no. 1, pp. 235-245, Feb. 1998.

[13] S. O. Rice, "Mathematical analysis of random noise," The Bell Labs Technical Journal, vol. 23, pp. 282-332, 1944.

[14] — "Mathematical analysis of random noise (concluded)," The Bell Labs Technical Journal, vol. 24, pp. 46$156,1945$.

[15] O. M. A.P. Prudnikov, Yu.A. Brychkov, Integrals and Series, Vol. 1: Elementary Functions. New York: Gordon and Breach Science Publishers, 1986.

[16] —_, Integrals and Series, Vol. 2: Special Functions. New York: Gordon and Breach Science Publishers, 1986.

[17] _ Integrals and Series, Vol. 3: More Special Functions. New York: Gordon and Breach Science Publishers, 1986.

[18] _ Integrals and Series, Vol. 4: Direct Laplace Transforms. New York: Gordon and Breach Science Publishers, 1992

[19] I. S. Gradshteyn and I. M. Ryzhik, Table of Integrals, Series and Products, 7th ed. Academic Press, 2007. 


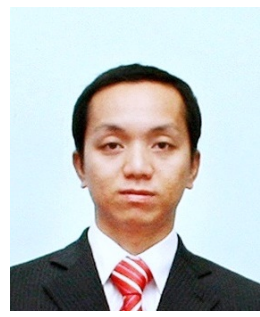

Viet-Ha Pham received the B.Sc.A., M.Sc., and Ph.D. degrees from Hanoi University of Technology, Hanoi, Vietnam; Pohang University of Science and Technology, Pohang, South Korea; and Laval University, Québec, QC, Canada, in 1997, 2003, and 2010. From Sep. 1997 to Aug. 2000, he is a lecturer at the Department of Computer Engineering, Hanoi University of Technology. He is currently a Postdoctoral Fellow at the Department of Electrical and Computer Engineering, University of Western Ontario, London, ON, Canada. His research interests include channel characterization and modeling, MIMO communications, channel prediction and adaptive transmission for broadband mobile wireless communications systems. He was a Technical Program Committee member for the 2008 IEEE ATC, 2010 IEEE VTC, 2011 IEEE ICC conferences and a reviewer for the IEEE Transactions on Broadcasting, IEEE Transactions on Vehicular Technology, IEEE Transactions on Signal Processing and IEEE Transactions on Wireless Communications. Dr. Pham is also an adjunct professor at Department of Computer Engineering, School of Information and Communications Technology, Hanoi University of Technology, Hanoi, Vietnam.

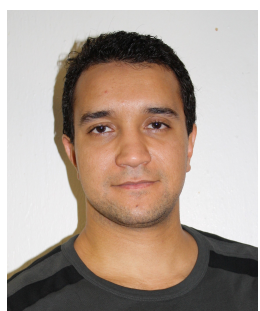

Mohamed Haj Taieb received his telecommunications engineer degree from the Higher School of Communication of Tunis (SUPŠCOM), Tunisia, in 2006. From 2006 to 2007 he worked in an IT services offshore company where he dealt with software development and network. He received his MSc degree from Laval University in 2009. His M.Sc research project is the evaluation of the wind turbines effects on the TV signals. He is currently pursuing his $\mathrm{Ph} . \mathrm{D}$. degree in electrical engineering at Laval University, Quebec, Canada with collaboration with the communication research center in Ottawa. His Ph.D. project deals with distributed video coding. His research interests include radio propagation, error correcting code, information theory, image and video processing.

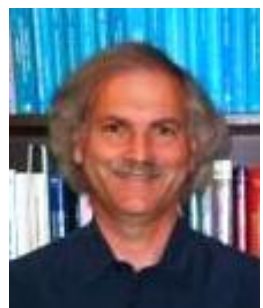

Jean-Yves Chouinard received the B.Sc.A., M.Sc., and Ph.D. degrees from Laval University, Laval, QC, Canada, in 1979, 1984, and 1987. He was a Postdoctoral Fellow at the Space and Radio Communication Division, Center National d'études des Télécommunications (CNET), Paris, France. From 1988 to 2002, J.-Y. Chouinard was a professor at the School of Information Technology and Engineering at the University of Ottawa. Since 2003 is with the Department of Electrical and Computer Engineering at Laval University.

$\mathrm{He}$ is the author or co-author of more than 160 journal, conference papers and technical reports. He is a co-inventor for 1 US patent and has 2 more US patents pendings. His research interests are communications theory and applications, broadband wireless systems, and advanced broadcast technologies. He is co-recipient with $\mathrm{F}$. Patenaude and J. Lodge of the 1999 Neal Shepherd Best Propagation Paper Award from the IEEE Vehicular Society. He also received the 2004 Signal Processing Best Paper Award with I. Sikaneta from the European Journal of Signal Processing. He is co-editor with P. Fortier and A. Gulliver of a book on information theory. He is co-author with S. Roy of a book chapter on software reconfigurable MIMO wireless communication systems and another book chapter with X. Wang on OFDM-based mobile broadcasting.

He was the Publications Chair for the IEEE International Symposium on Information Theory (ISIT'2008) and a General Co-chair for the Canadian Workshop on Information Theory (CWIT'95). He is a Technical Program Co-chair for the upcoming 2012 Vehicular Technology Conference (VTC'2012 Fall) in Quebec city, Canada. He is also a Director of the Canadian Society of Information Theory (CSIT). $\mathrm{He}$ is an Associate Editor for the IEEE Transactions on Broadcasting.

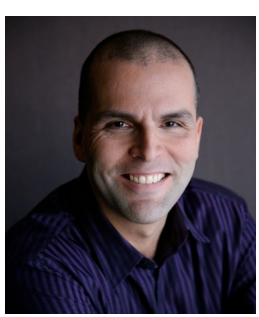

Sébastien Roy received the B.Sc. and M.Sc. degrees in electrical engineering from Laval University, Québec, QC, Canada, in 1991 and 1993, respectively, and the Ph.D. degree from Carleton University, Ottawa, ON, Canada, in 2000. He is currently Full Professor with the Department of Electrical and Computer Engineering, Laval University, where he is pursuing research in the system-level and implementation aspects of signal processing for communications as well as space-time processing and space-time coding. From 2000 to 2002, he was a Natural Sciences and Engineering Research Council of Canada (NSERC) Postdoctoral Fellow at Laval University. He has also been active in industrial consulting with companies such as InterDigital and MacDonald Dettwiller, and was involved in the organization of several international conferences. In 2007 and 2009, he was an invited professor at lcole Nationale Suprieure de Sciences Appliques et de Technologie (ENSSAT), Lannion, France. He received 5 teaching awards and in 2007 received the award for excellence in technology transfer from the strategic network on Systems and Technologies for Advanced Communications (SYTAcom). Dr. Roy was also bestowed the award for Post-Graduate Research Excellence from the Canadian Institute for Telecommunications Research in 2000.

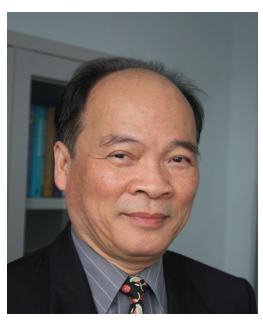

Huu-Tue Huynh was born in Hue, Viet Nam. He received the Sc.D. degree in 1972 from Laval University, Canada, where from 1969 to 2004 he was a faculty member of the Department of Electrical and Computer Engineering. In 2004, he left Laval University to become Chairman of the Department of Data Processing at The College of Technology of the Vietnam National University, Hanoi. Since 2007 he has been President of Bac Ha International University, Vietnam. He was an Invited Guest at The AT\&T Information Systems in Neptune, N.J. in 1984 and has been invited to give lectures at several Universities in Europe, America as well as in Asia. Professor Huynh is author and coauthor of two books and more than two hundred papers in Information Processing. He has served as Consultant to a number of Canadian Government Agencies and Industries. His research interests cover stochastic simulation techniques, information processing, fast algorithms and architectures with applications to digital communications. 